\title{
Python - Based Image Processing
}

\author{
${ }^{1}$ Mrs. Asha K H, ${ }^{2}$ Manjunathswamy B E, ${ }^{3}$ Mrs. Chaithra A S \\ Don Bosco Institute of Technology, Bangalore, India
}

\begin{abstract}
.
The main goal of the Image Process project is to extract important information from photographs. The machine may produce a description, interpretation, and comprehension of the scene based on this extracted data. The main goal of image processing is to transform photos in the desired way. This technique allows users to obtain the text of picture processing printing processes and to save the data to disc in a variety of formats. In other terms, image processing is the process of neutering and analyzing graphical information in photographs. In our lives, we frequently come across many types of image processing. The clearest example of image processing in our lives is our brain's perceiving of visuals. Once we perceive pictures with our eyes, the process takes relatively little time
\end{abstract}

Keywords. Image acquisition, Image normalization, Image segmentation, resizing the image, Wavelet Transform.

\section{Introduction}

Image recognition methods are a signal dispensation in which the input is an image, such as a video frame or photograph, and the output is an image or attributes associated with that image. An image method system, in general, treats video as two-dimensional signals that are then exposed to signal procedures that have been pre-programmed. It's one of the most rapidly evolving technologies, with applications in a wide range of fields. There are sections on imaging methods, core analysis, engineering, and topic.

The process of converting an image to digital form and then performing operations on it to improve the image or extract pertinent data is known as image processing. It's a signal distribution method in which the input is an image (such as a video frame or a photograph) and the output is an image or image-related attributes. Images are typically treated as two-dimensional signals that are subjected to pre-determined signal processing techniques by an Image Processing system. It is one of the most rapidly developing technologies today, with applications in a wide range of industries. In both engineering and computer science, image processing is an important study issue.[1,5].The three phases that make up image processing are as follows:

- Using picture acquisition tools to import the image

- Examining and modifying the image

- Output that may include a changed image or a report based on image analysis.

- Edge detection is a low-level image processing method.

Low-level image processing algorithms include :

- Detection of the edges

- Customer segmentation.

- Classification;

- Detection and matching of features.

\section{LiteratureSurvey}

Stefan van der Walt, Johannes L. Schonberger ,Juan Nunez-Iglesias, Franc sois Boulogne, Joshua D. Warner, Neil Yager, Emmanuelle Gouillart, Tony Yu et al..[2 ]expressed asA devoted group of volunteers created scikit-image, a suite of image processing algorithms written in Python and released under the BSD Open Source license. Python's growing popularity as a scientific programming language, along with the expanding availability of a large ecosystem of supporting tools, makes it an ideal environment for creating 
an image processing toolkit.

Yurong Guan1, Fei Zhou2, Jing Zhou et al..[3] expressed as In the practise of digital image processing, the relevant experimental programming language should be expanded. Python should be the testing language of choice. It is easier to grasp the language itself, letting students to focus their energy and time on envisioning solutions to problems, thereby increasing their desire to learn and improve their practical abilities. In recent years, Python has grown in popularity in the field of digital image processing. The use of language in mathematical modelling has received a lot of attention, as have various big data competitions.Based on a study and analysis of the PIL library, there is still room for more research and development in areas such as image and graphics contrast processing, graphics image storage, and graphics image rendering. It also covers picture filtering technology, outline image contour, relief style, image edge, and more.

Muhammad ArifRidoy et al..[ 4] expressed as It has seen significant growth in both reception and dedication in recent years, and the group is keen to collaborate with others to help it grow even more, and to establish it as the definitive Python image processing library. Scikit-image performs a wide range of image processing calculations using a simple interface that works well with both $2 \mathrm{D}$ and $3 \mathrm{D}$ images. It is completely integrated into the Scientific Python environment, making it compatible with perception libraries and other data preparation tools. Scikit-image has grown tremendously since its inception in 2009, both in terms of clients and added features. Despite the growing number of logical groups that use scikit-image to prepare images of various X-beam modalities, area-specific instruments are now relying on scikit-image to expand their capabilities.

\section{Methodology}

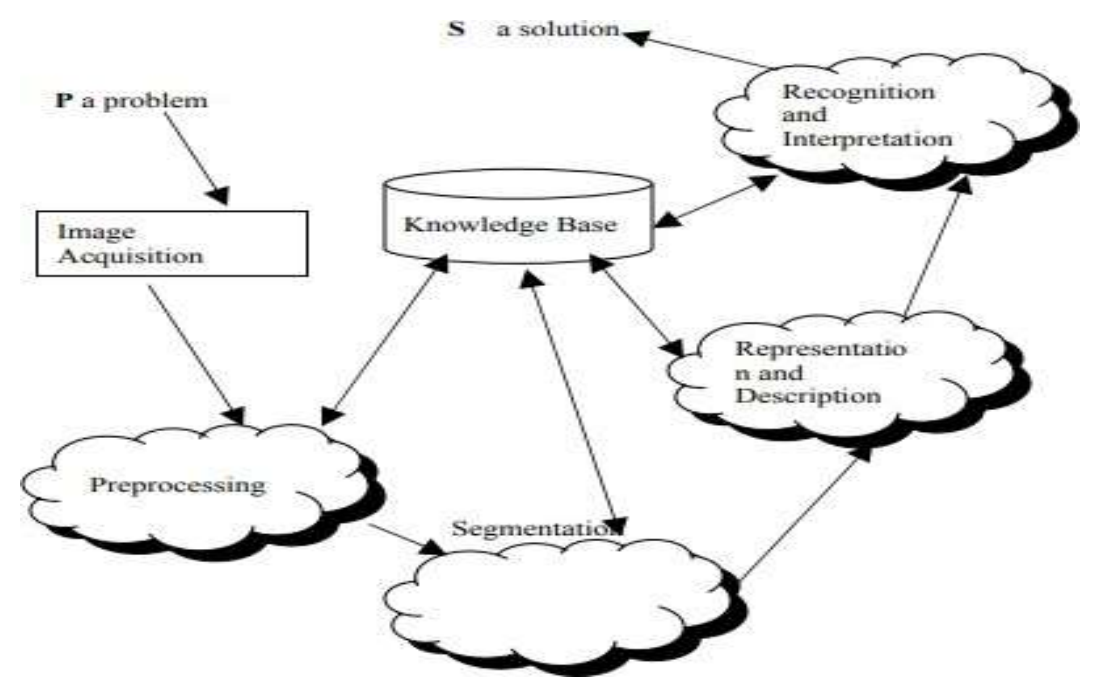

Fig 1. Image processing in action

- Acquisition of Image - Image processing in action A photograph is captured and digitized by a sensor device (such as a monochrome or colour TV camera). An analog-to-digital device translates the output of the camera or sensor element if it isn't already in digital format [6].

- Recognition and Interpretation- Recognition is the process of assigning a label to an item while taking into account the information offered by its descriptors. Interpretation is a distribution in a nursing ensemble that aims to connect recognized objects.

- Segmentation- It's a catch-all term for a number of ways for breaking down an image into its component pieces. The process of breaking down the information contained in an image into segments is known as segmentation.smaller pieces that can be used for a variety ofreasons.

- Representation and Outline - Through representation and outline, data is turned into a format that is suitable for the popularity process.

- Domain of Information: The drag domain that describes the areas of a picture where the knowledge of 
interest is assumed to be set is known as cognitive content. It aids in the search's narrowing..

- Detection of edge- It's a catch-all term for a collection of procedures and methodologies for maintaining a photograph and creating an image drawing. Thelines indicated changes in values such as plane cross sections, plane crossings, textures, lines, and colours, as well as shading and texture alterations.

- Image Compression- Massive volumes of data are contained in electronic images, necessitating the use of data transfer networks capable of handling large amounts of data. The requirement for temporal and abstraction resolution, the number of photos per second, and hence the range of grey levels is influenced by the quality of a photograph.

- Real time image process- Two different real-time processing techniques have been proposed. One option is to develop dedicated hardware that enables the process to run in real time. The recommended technique, on the other hand, is to increase the capability of both the software package and, as a result, the hardware, while minimizing process and machine requirements.

\section{Results}

The proposed implementation demonstrates an image processing technique that involves taking a test image and then normalizing, resizing, and padding it.The image recognition representation and output displayed after implementing the code using Python Language in Visual Studio Code are shown below.
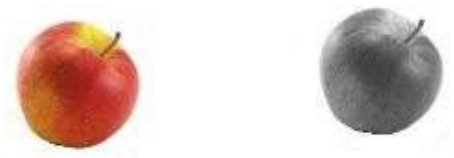

Normalized image

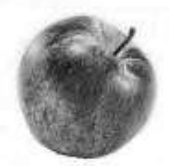

Contrast enhancement
Gray-Scale image

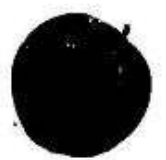

Binary image

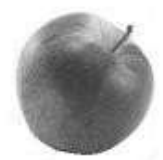

Resize image

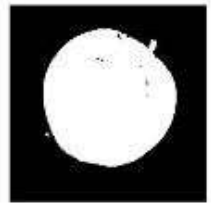

Complemented Binary image

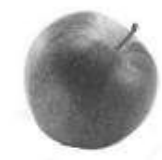

Noise removal

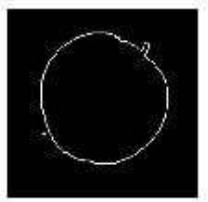

Boundary image

Fig 2. Image Processing Representation
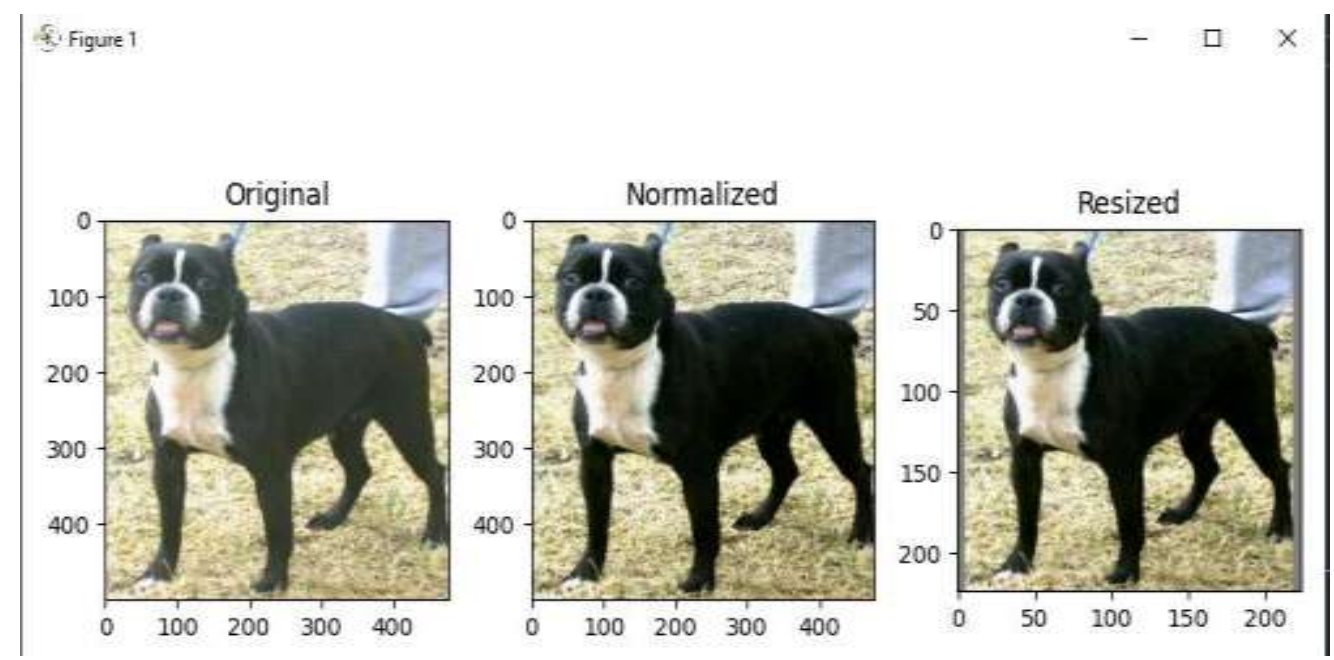

Fig 3. Image processing output

\section{Conclusion}

It's a significant study with a lot of ramifications nowadays because it's tied to advanced science and 
technology. Technological advancements have opened up a plethora of possibilities for the Vision System and Image Process. The increasing trend will almost certainly continue in the future. Image processing is used to recognize photographs in mill floor quality assurance systems, image sweetening, and satellite intelligence systems.Using image processing techniques, we can sharpen photos, distinguish them to create a more effective graphic display, lower the amount of memory required for storing image definite motion, and soon, reduce the amount of memory necessary for storing image certain motion. As a consequence of the preceding discussion, we can conclude that this subject has far more advantages than disadvantages, and as a result, it is advantageous in a wide range of sectors.

\section{References}

1. Nour Eddine ALAA and Ismail Zine El Abidne, Introcduction To Image Processing With Python, March 5, Cadi Ayyad University,2021,77p

2. Stefan van der Walt, Johannes L. Schonberger ,Juan Nunez-Iglesias, Franc sois Boulogne , Joshua D. Warner, Neil Yager, Emmanuelle Gouillart, Tony Yu ,scikit-image: Image processing in Python, arXiv:1407.6245v1 [cs.MS] 23 Jul 2014, DOI: 10.7717/peerj.453 • Source: arXiv.

3. Yurong Guan1, Fei Zhou2, Jing Zhou, Research and Practice of Image Processing Based on Python, CISAT 2019 , IOP Publishing Journal of Physics: Conference Series 1345 (2019) 022018 doi:10.1088/1742-6596/1345/2/022018

4. Muhammad ArifRidoy, Image processing in Python, International Journal of Scientific \& Engineering Research, March-2018, Volume 9, Issue 3, ISSN 2229-5518,p1386- 1391

5. D. Sri Shreya ,Digital Image Processing And Recognition Using Python, International Journal of Engineering Applied Sciences and Technology, , 2021 Vol. 5, Issue 10, ISSN No. 2455-2143, Pages 319-322

6. Dr.PL. Chithra, P.Bhavani,A Study On Various Image Processing Techniques, International Journal of Emerging Technology and Innovative Engineering Volume 5, Issue 5, May 2019 (ISSN: 2394 6598) 